\title{
The Sodium Laser Guide Star System of ALFA
}

\author{
Andreas Quirrenbach, Wolfgang Hackenberg, Hans-Christoph Holstenberg \\ and Norbert Wilnhammer \\ Max-Planck-Institut für Extraterrestrische Physik Giessenbachstraße \\ D-85478 Garching Germany
}

\begin{abstract}
ALFA is an adaptive optics system with laser guide star for the $3.5 \mathrm{~m}$ telescope on Calar Alto, Spain. ALFA has been developed jointly by the Max-Planck-Institutes for Astronomy (MPIA, Heidelberg) and for Extraterrestrial Physics (MPE, Garching). All core components of ALFA were installed at the telescope in the course of 1996; the system is currently undergoing integration and testing. ALFA uses a continuous-wave dye laser pumped by an argon ion laser to create an artificial guide star in the mesospheric sodium layer. The laser is stabilized to the sodium $\mathrm{D}_{2}$ line at $589 \mathrm{~nm}$ with a sodium vapor cell. In normal operation the frequency is locked to the Lamb dip in the absorption cell, but it is also possible to scan the laser under computer control over several $\mathrm{GHz}$ for diagnostic purposes. The dye laser has been optimized for stable operation at high power output; $3.75 \mathrm{~W}$ are regularly achieved in single-mode operation. The laser system is located in the coudé room of the telescope; the laser beam is transmitted through the coudé path to a $50 \mathrm{~cm}$ beam expansion telescope attached to the mirror cell of the $3.5 \mathrm{~m}$ telescope. The first measurements of the sodium spot created with the ALFA laser were made in 1996 with the guide camera of the $3.5 \mathrm{~m}$ telescope and with a $1.23 \mathrm{~m}$ telescope located $276 \mathrm{~m}$ away.
\end{abstract}

Keywords: adaptive optics, laser guide stars, sodium lasers, cw dye lasers, laser beam projection

\section{INTRODUCTION}

ALFA (Adapative optics with Laser guide star For Astronomy) is a joint project of the Max-Planck-Institutes for Astronomy (MPIA, Heidelberg) and for Extraterrestrial Physics (MPE, Garching). ALFA is currently undergoing integration and testing at the $3.5 \mathrm{~m}$ telescope at the German-Spanish Center for Astronomy on Calar Alto, Spain. The aim of the ALFA project is to provide nearly diffraction-limited imaging in the $\mathrm{H}$ and $\mathrm{K}$ near-infrared bands under median seeing conditions, and in the $\mathrm{J}$ band under favorable conditions. A sodium guide star system has been included in the design and construction from the beginning. Here we summarize the most important features of ALFA's adaptive optics bench, and then describe the sodium laser and laser launch systems. A brief preliminary report on the status of ALFA in 1996 was given by Quirrenbach. ${ }^{1}$

\section{THE ADAPTIVE OPTICS BENCH}

ALFA uses a 97-actuator deformable mirror with a continuous face sheet procured from Xinetics. The wavefront is measured with a Shack-Hartmann device; a number of lenslet arrays are mounted on a filter wheel, so that different pupil geometries can be chosen. This provides flexibility to optimize the performance depending on the brightness of the guide star, and under varying seeing conditions. The wavefront sensor is based on a fast CCD chip developed by Lincoln Labs, which can be read at frame rates between $100 \mathrm{~Hz}$ and $1200 \mathrm{~Hz}$. The read noise of ALFA's wavefront sensor camera has been measured to be $6 \mathrm{e}^{-}$at a frame rate of $1200 \mathrm{~Hz}$. The hardware and software for wavefront reconstruction and control of the deformable mirror have been developed by Adaptive Optics Associates. An independent CCD camera measures the image motion (tip/tilt) of a guide star. The WFS and tip/tilt cameras can either look at the same star (for operation with a natural guide star), or at separate objects (for operation with a laser guide star).

ALFA is mounted at the Cassegrain focus of the $3.5 \mathrm{~m}$ telescope, between the telescope itself and the science instrument. The number of optical elements in the light path is as small as possible (namely 5), to avoid light loss and to keep the additional thermal background low. The input $\mathrm{f} / 10$ beam from the telescope is reflected by an active mirror, which corrects the tip/tilt (image motion), onto an off-axis parabola, which produces an image of the pupil on the deformable mirror. Another off-axis parabola produces the f/25 output beam, which is split into visible (used 
for wavefront sensing) and IR (used by the scientific instrument) by a dichroic beam splitter. The tip-tilt mirror and the dichroic are motorized and can be moved out of the beam. All optical elements of ALFA are thus bypassed, and the beam to the scientific instrument changes from $\mathrm{f} / 25$ to $\mathrm{f} / 10$, giving a 2.5 times larger pixel scale. This allows for quick changes of the observing program from high-resolution observing to wide-field imaging when the seeing gets too bad for efficient use of the adaptive optics. ALFA will be used with OMEGA, an infrared camera based on a Rockwell detector with $1024 \times 1024$ pixels currently under development at MPIA, and with 3D, an infrared integral field spectrometer built at MPE. ${ }^{2}$

During the first engineering runs of ALFA, the adaptive optics loop was closed on stars with brightness down to $m_{V} \sim 10.5$, mostly in poor seeing conditions $\left(\gtrsim 1 !^{\prime \prime} 2\right)$. The FWHM of the corrected images $\left(0{ }^{\prime \prime} 15\right.$ in $\mathrm{K}$ band) was close to the diffraction limit of the telescope, and the Strehl number was improved by typical factors of 10. Further tests in good seeing conditions are needed to determine the limiting magnitude and image quality of ALFA.

\section{THE LASER SYSTEM}

ALFA's laser system is based on a Coherent Model 899-21 dye ring laser using Rhodamine 6G, noncollinearly pumped by an argon ion laser with $25 \mathrm{~W}$ multiline output power (Coherent Innova 400).

The basic resonator of the dye laser is a figure-8-shaped ring, formed with 3 spherical mirrors and one flat output coupler, all mounted to a solid Invar rod. There are two beam waists: one is optically pumped at the dye jet; the other is an auxiliary waist for intracavity experiments. A curved mirror focuses the pump light into the free-flowing dye jet stream. Two curved mirrors match the beam diameter of the dye laser cavity mode to the focused pump spot. The dye jet is a nearly rectangular ribbon with a thickness of $0.3 \mathrm{~mm}$, and it is oriented at the Brewster angle relatively to the laser cavity mode and near Brewster's angle to the pump beam, in order to minimize reflection loss and to prevent unwanted polarization effects. The ring design was chosen because unidirectional ring dye lasers with traveling wave fields have advantages over bidirectional or standing-wave dye lasers because of more efficient use of the available gain in the active medium and better spectral characteristics. An optical diode consisting of a Faraday rotator and a quartz plate as optical derotator ensure that the laser oscillates as a travelling wave. A glass rhomb mounted near the auxiliary waist at Brewster's angle compensates for astigmatism caused by off-axis reflection from the three spherical mirrors. This improves the mode quality and the spectral properties of the laser.

The oscillation mode of the laser is selected by a three-plate birefringent filter followed by electronically controlled thin and thick etalons. To achieve tunability within the mode separation, the cavity length can be continously changed by rotating a single galvo-driven Brewster plate mounted at the vertex of the ring. This position minimizes output power modulation and beam movement during a frequency scan. For active frequency stabilization a part of the dye laser output is sent into an external reference cavity. The error signal from the reference cavity controls the scanning Brewster plate and a piezo-mounted folding mirror for fast cavity length adjustments. For absolute wavelength tuning and long-term frequency stabilization the reference cavity is locked to the Lamb dip in the saturated fluorescence signal of the sodium $\mathrm{D}_{2}$ line from a laboratory sodium cell.

\section{OPTIMIZATION OF THE DYE LASER}

In addition to the optimization of the noncollinear pumping geometry and the output transmission, the main improvement of the commercial dye laser was achieved by optimizing the optical quality of the active laser medium at high pump powers.

The optimum flow velocity at a given viscosity of the dye solvent and jet thickness was determined both by analyzing the jet thickness fluctuations with the help of an interferometer and directly by measuring the laser performance (output power, mode quality and linewidth). The jet nozzle used in the experiment has a rectangular flow cross section with a $6 \mathrm{~mm}$ diameter feed hose connection smoothly decreasing to the nozzle channel, which is $25 \mathrm{~mm}$ long. The nozzle is constructed from two stainless steel plates with highly polished dye contact surfaces. The jet thickness and height are controlled by precision spacers. In order to avoid concentration quenching the optimum jet thickness (at optimum flow velocity) was found to be around $0.3 \mathrm{~mm}$ for the case of an ethylene glycol solution with the dye absorption set to $75 \%$. It is slightly smaller for a water-based dye solution with a viscosity-raising additive (20\% Ammonyx LO, 5\% polyvinyl alcohol) and $80 \%$ pump light absorption. The optimum jet height for both solvents was found to be $6 \mathrm{~mm}$. To achieve the required high flow velocities a high pressure, a dye circulation 
Figure 1. Schematic drawing of the laser system located in the coudé room of the $3.5 \mathrm{~m}$ telescope. The $\mathrm{Ar}^{+} \mathrm{pump}$ laser, the dye laser, the sodium reference cell, as well as a beam pre-expander and beam diagnostics are all mounted on a single optical table.

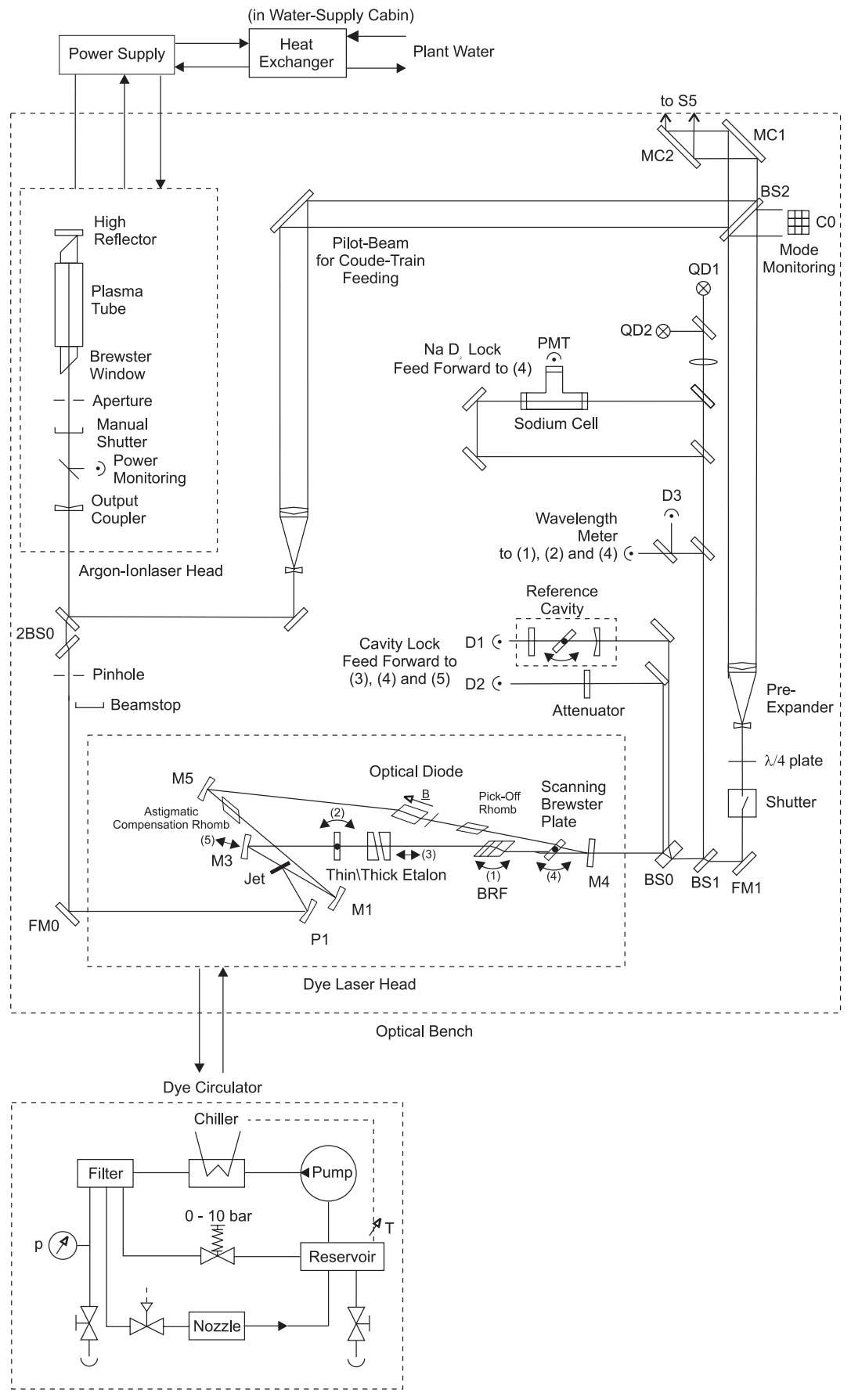


Table 1. Optimized parameters of the dye jet for two different dye solutions.

\begin{tabular}{|l|c|c|}
\hline & Jet $\sharp 1$ & Jet $\sharp 2$ \\
\hline \hline Solvent & Ethylene glycol & $\begin{array}{c}75 \% \text { water } \\
+20 \% \text { lauryl amine } \\
+5 \% \text { polyvinyl glycol }\end{array}$ \\
\hline Temperature & $23^{\circ} \mathrm{C}$ & $8^{\circ} \mathrm{C}$ \\
\hline Jet Thickness / Height & $0.3 / 6 \mathrm{~mm}$ & $0.3 / 6 \mathrm{~mm}$ \\
\hline Flow Velocity & $22 \mathrm{~m} / \mathrm{s}$ & $14 \mathrm{~m} / \mathrm{s}$ \\
\hline Rh6G Absorption & $75 \%$ & $80 \%$ \\
\hline Pump Focus Spot- $\varnothing$ & $25 \mu \mathrm{m}$ & $20 \mu \mathrm{m}$ \\
\hline
\end{tabular}

system was constructed out of material compatible with Rh6G. Attention was paid to effective damping of vibrations. In the case of ethylene glycol, the temperature of the solution has to be stabilized to about $23^{\circ} \mathrm{C}$ to achieve the optimum flow velocity, whereas the water-based solution has to be cooled below $10^{\circ} \mathrm{C}$.

With the water-based dye solution negligible thermal distortion of the optical qualitiy of the dye jet occurs up to argon-ion multiline pump powers of $30 \mathrm{~W}$, with a pump conversion efficency of about $18 \%$. With this dye solution, up to $5.5 \mathrm{~W}$ single-line output could be achieved. Even higher output power is probably possible with the use of a triplet quencher. The dye laser output power in the case of an ethylene glycol solution was found to be limited to about $4.5 \mathrm{~W}$ at $30 \mathrm{~W}$ pump if a near diffraction-limited output mode quality is required. A summary of the dye jet parameters is given in Table 1. The dye laser output power is given as a function of the pump power in Figure 2.

The laser is now operated at the Calar Alto observatory. To keep the maintenance at a minimum, an ethylene glycol dye solution is currently used; an output power of $3.75 \mathrm{~W}$ is routinely achieved without extensive optimization.

\section{BEAM PROJECTION}

The lasers are located in the coudé room of the $3.5 \mathrm{~m}$ telescope, and the coudé path of the telescope is used for the beam-delivery system, shown schematically in Figure 3. The mirrors S4 and S5 are part of the coudé train of the telescope; all other mirrors were installed for the laser system. The mirrors MC1 and MC2, located on the laser bench, are used to feed the collimated $20 \mathrm{~mm}$ diameter beam into the coudé train. S5 is located on the right ascension axis of the telescope, it is fully steerable to follow the telescope motion. S5 is driven by the telescope control system, which operates independently of the laser control system. The mirrors S4 and MT1 are located on the declination axis. MT1, at a position above and just off the edge of the primary mirror, picks the beam off the coudé path; MT1 through MT4 direct it to the laser launch telescope. The launch telescope is a reflective system with $0.5 \mathrm{~m}$ diameter and an actively controlled secondary mirror for tip-tilt correction and focusing. It is mounted to the mirror cell of the $3.5 \mathrm{~m}$ telescope, at approximately $1 \mathrm{~m}$ distance from the primary mirror. A breadboard at the entry of the launch telescope provides space for beam diagnostics, such as power meter and beam profiler, which can be inserted into the laser beam during alignment and maintenance.

Three breadboards are installed along the beam path, which provide space for the installation of beam diagnostics and additional components required for beam alignment and control. First tests have shown that a set of actively controlled beam steering mirrors are required to keep the laser beam aligned during telescope slews. This is achieved by two separate control loops for the lower and upper sections of the beam-relay system. For minimal interaction with the dye laser beam, a set of separate guide lasers, which are focused on $400 \mathrm{~mm}^{2}$-sized position sensitive devices (lateral-effect photodiodes by SiTek), are used. For the upper section two laser diodes are installed close to the dye laser beam at the entry of the launch telescope. The beams of these laser diodes are directed almost parallel to the dye laser beam. For the lower section a small fraction of the $\mathrm{Ar}^{+}$pump laser energy is used for alignment purposes. Coated beamsplitters (BS2 and BS3) with a low reflectivity $(<0.4 \%)$ are used to get the $\mathrm{Ar}^{+}$guide beam on and off the dye laser axis. The control loops are designed to operate at $100 \mathrm{~Hz}$, controlling fast, high precision dc motors at the MT1, MT3, and MT4 positions, and a tip/tilt piezo stage for MC2. The whole system is controlled by a networked VMEbus computer running EPICS for near-realtime communication with the telescope control and AO systems. The graphical user interface has been implemented on UNIX workstations. While sensors and actuators 
Figure 2. Dye laser power as a function of argon ion pump power, for the two dye solutions desribed in Table 1. The dotted lines are linear extrapolations from the output at low pump power.

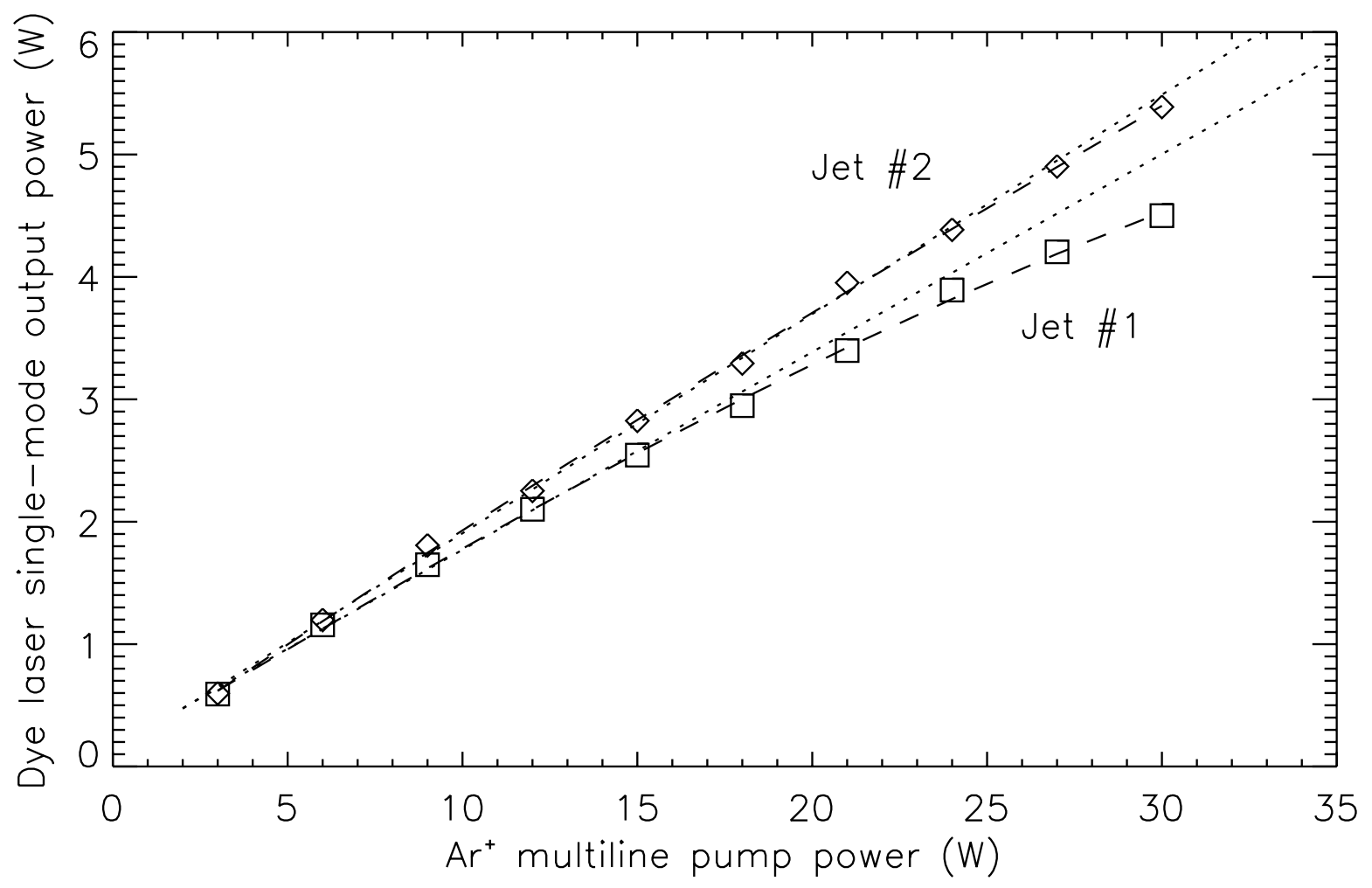

allow for $\mu \mathrm{m}$ resolution and accuracy, the precision of this system is still limited by seeing along the path of the laser beam, which passes through several areas with large temperature gradients. By far the largest temperature difference is between the coudé lab and the dome, but there are also noticeable sources of heat in the yoke of the telescope. Windows and light tubes are used to ensure acceptable beam quality, and additional baffles will further improve the system performance.

Continuous reliable operation of the electronics and the mechanical components at temperatures between $0^{\circ} \mathrm{C}$ and $20^{\circ} \mathrm{C}$ require the use of high-quality components and special care in evaluating the environmental specifications of these devices. Furthermore, some components are located in locations that are not conveniently accessible for maintanance, such as the yoke. Safety issues have been addressed by encapsulating the laser beam and all major components in light tubes and cases. These measures are also required for reducing the stray light originating from this system, which is particularly important in the area close to the primary mirror.

\section{FIRST RESULTS AND FUTURE PROSPECTS}

The ALFA laser system has been used to generate sodium spots during several test runs at the $3.5 \mathrm{~m}$ telescope. An image from the first run taken with the telescope guide camera is shown in Figure 4 . During this run the beam quality was poor due to excessive turbulence in one of the beam pipes; therefore the sodium spot was about $5^{\prime \prime}$ large. After corrections of the "pipe seeing" problem, we have obtained sodium spots with 3 " FWHM in 2 " seeing. Further tests in good seeing are required to determine whether the spot size is limited by external seeing or internal turbulence. 
Figure 3. Schematic drawing of the beam projection system.

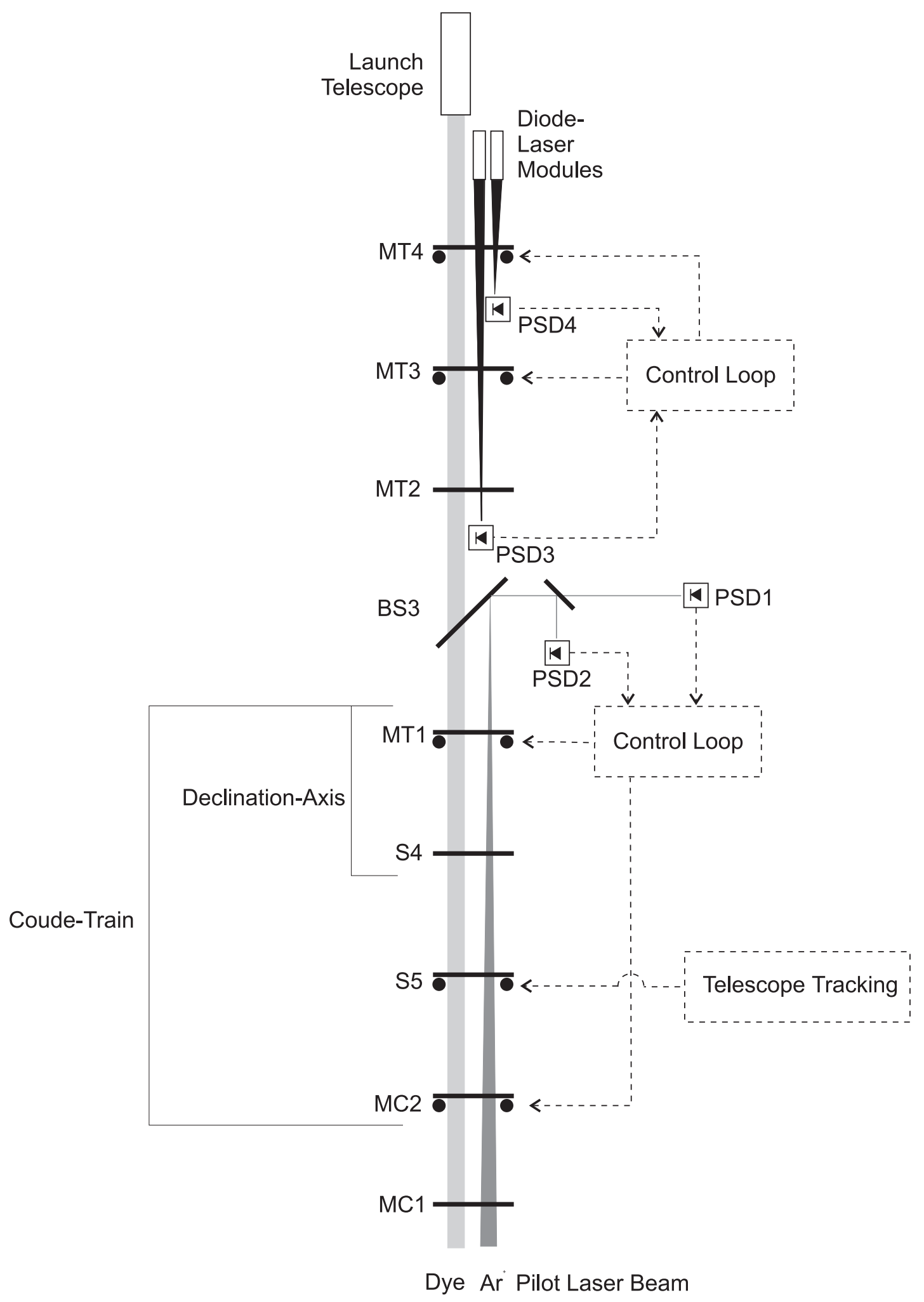


Figure 4. First image of the spot generated with the ALFA laser in the mesospheric sodium layer. The image was taken with the guide camera of the telescope. The Rayleigh cone is seen in the bottom left corner, the sodium spot is at the tip of the cone. The telescope was focused to the altitude of the sodium layer $(\sim 90 \mathrm{~km})$; the image of a star therefore appears defocused.

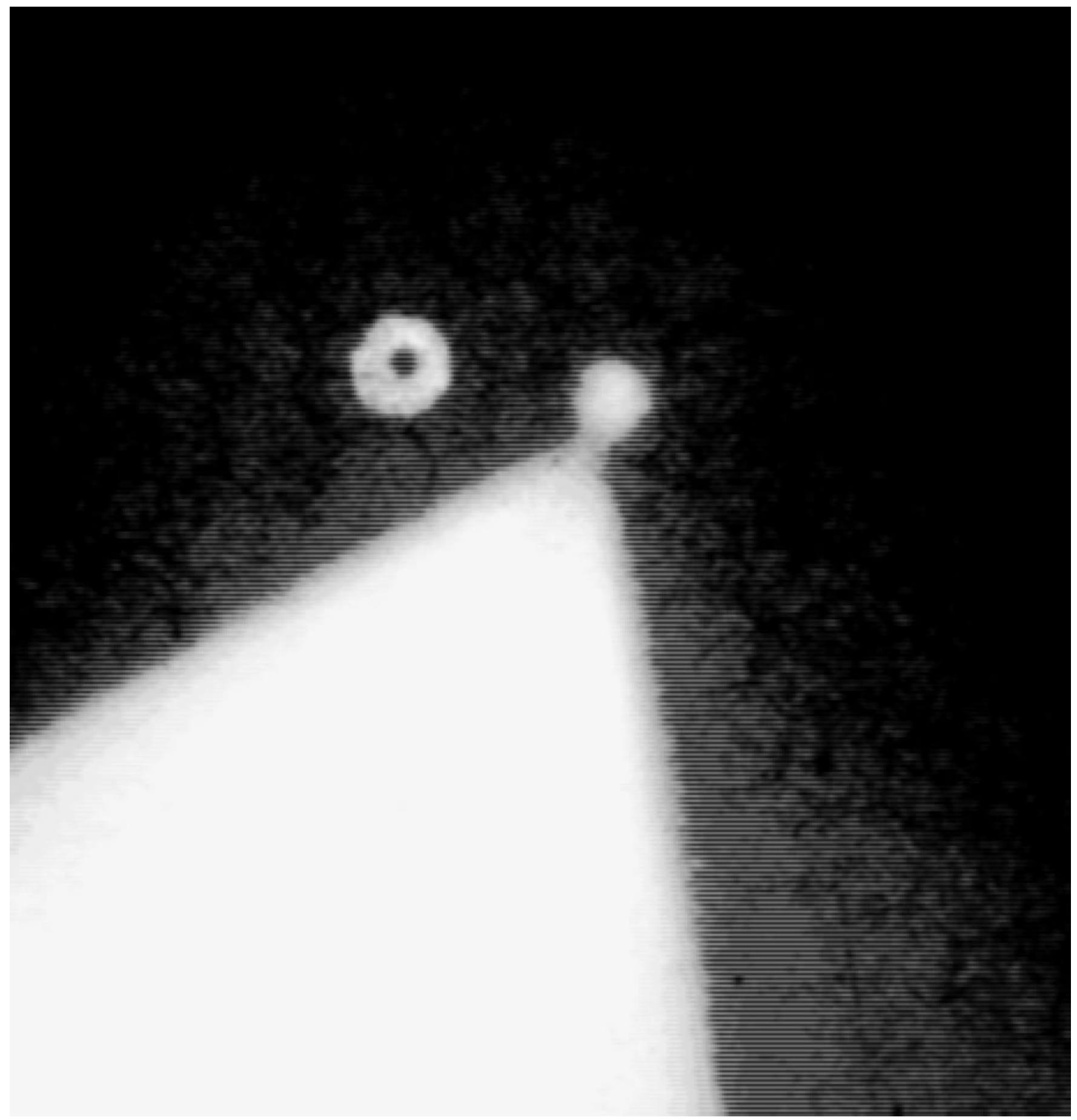


Additional images of the sodium spot were taken with a $1.23 \mathrm{~m}$ telescope located $276 \mathrm{~m}$ from the $3.5 \mathrm{~m}$ dome. Seen from this distance, the elongation of the sodium spot is $\sim 140^{\prime \prime}$. It is therefore fairly straightforward to use its brightness profile to derive the density profile of the mesospheric sodium layer. This information is required for correct focusing of the laser guide star system.

Due to non-photometric conditions during most of the test nights, we have only once been able to obtain reliable photometry of the sodium spot. On that date we obtained a return signal from the sodium layer with an equivalent magnitude of $m_{V} \sim 9.5$. This is comparable to the brightness of the faintest stars that have been used to operate the ALFA adaptive optics system. We will therefore try to close the AO control loop with a sodium guide star in the near future. It will probably be possible to further enhance the sodium return signal. The laser power (currently $3.75 \mathrm{~W}$ for regular operations can be increased by using a water-based dye solution and triplet quenchers, as described above. Furthermore, we currently do not control the polarization state of the laser beam. The dye laser itself generates a linearly polarized beam, but there are a number of oblique reflections in the beam path, and the angle of incidence of some mirrors changes with the telescope pointing. We intend to use circular polarization in the future, which maximizes the sodium return signal. Together with improvements in the user interfaces, these measures should allow regular operation of the ALFA adaptive optics and laser guide star systems for astronomical purposes and as a testbed for the next generation of laser guide star systems.

\section{ACKNOWLEDGEMENTS}

We thank our colleagues at MPIA and on Calar Alto for their collaboration on the ALFA project. We have benefitted enormously from discussions with Drs. R. Angel, H. Friedman, R. Fugate, T. Jeys, E. Kibblewhite, C. Max, D. Murphy, and C. Townes. The design of the ALFA laser system would not have been possible without their suggestions.

\section{REFERENCES}

1. Quirrenbach, A., "Adaptive optics at MPE: astronomical results and future plans", in Adaptive optics, 1996 Technical digest series Vol. 13. Optical Society of America, p. 166-167, 1996

2. Weitzel, L., Krabbe, A., Kroker, H., Thatte, N., Tacconi-Garman, L.E., Cameron, M., \& Genzel, R., "3D: The next generation near-infrared imaging spectrometer", A\&AS 119, 531-546, 1996 
Figure 5. Image of the sodium spot, taken with the $1.23 \mathrm{~m}$ telescope, which is at a distance of $276 \mathrm{~m}$ from the $3.5 \mathrm{~m}$ telescope. The elongation of the spot is about $140^{\prime \prime}$ when seen from this distance. Images like this one are used to derive density profiles of the sodium layer.

a

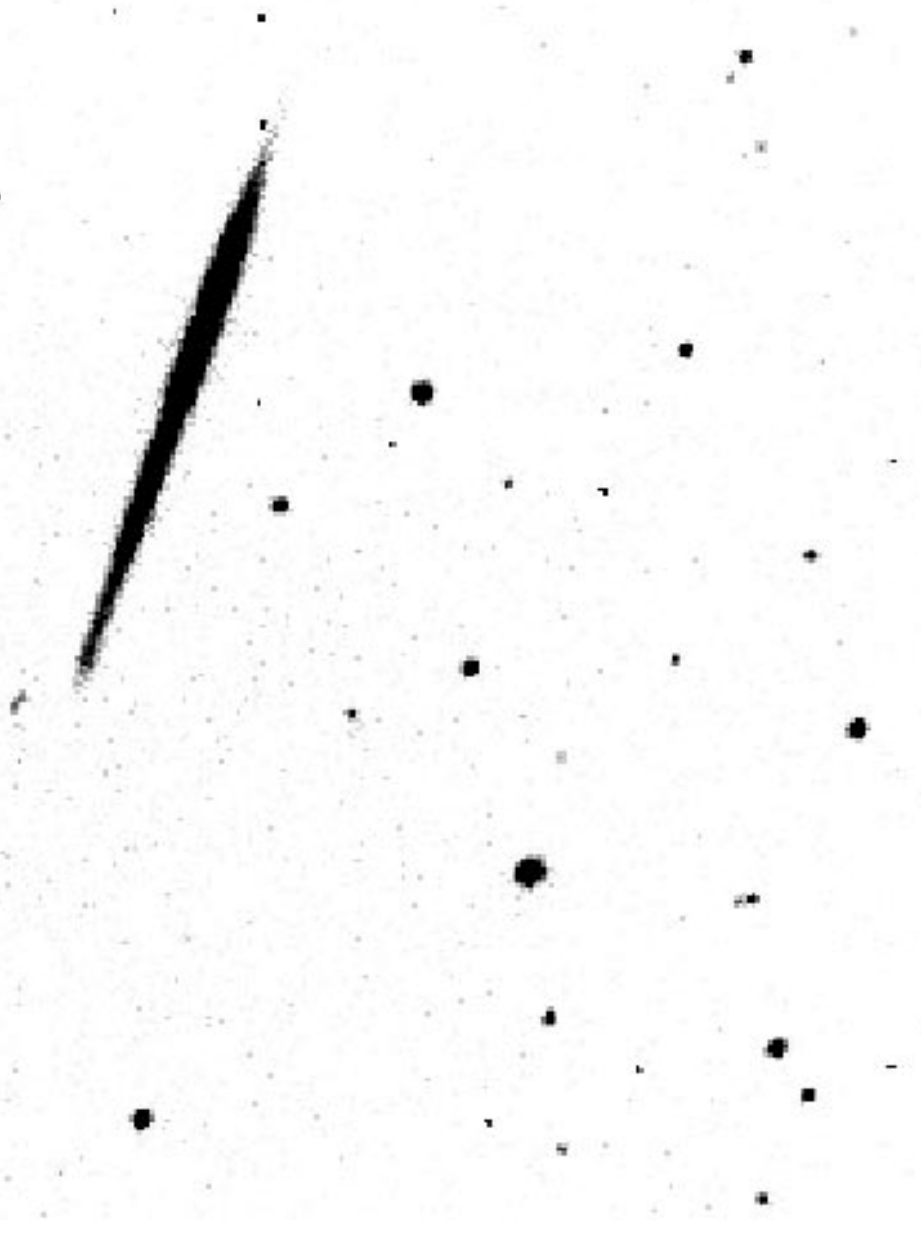

